\title{
Detection and identification of pathogenic dermatophytes using multiplex real-time PCR assays
}

Franziska Wittig ${ }^{1}$, Silke Uhrlaß¹, Giel Gaajetaan², Gijs Dingemans², Constanze Krüger ${ }^{1}$, Pietro Nenoff ${ }^{1}$

${ }^{1}$ Laboratory of Medical Microbiology, Partnership Dr. C. Krüger \& Prof. P. Nenoff, Moelbiser Hauptstrasse 8, 04571

Roetha OT Moelbis, Germany

2 PathoNostics B.V., Randwycksingel 45, 6229 EG Maastricht, the Netherlands

Introduction

Fungal infections of nails, skin and hair (tinea) caused by dermatophytes such as Trichophyton (T.) spp., Microsporum (M.) spp. or Epidermophyton (E.) spp. are one of the most common human infection in the world (White et al.). As the epidemiology varies between different dermatophyte species a specific diagnosis plays an important role to ensure a targeted therapy. In order to evaluate the DermaGenius ${ }^{\circledR}$ multiplex real-time PCR assays (PathoNostics), qPCR results were compared to routine diagnostic methods including fungal culture detection, microscopy of native specimens and a standard PCR-ELISA assay as well as Sanger sequencing.

Tab. 1: Clinically isolated dermatophytes.

\begin{tabular}{|c|c|c|c|c|c|}
\hline \multirow[b]{2}{*}{ \# } & \multirow{2}{*}{$\begin{array}{c}\text { Fungus } \\
\text { (PCR-ELISA } \\
\text { Seq.) }\end{array}$} & \multicolumn{2}{|c|}{ Microscopy } & \multirow[b]{2}{*}{ qPCR result } & \multirow[b]{2}{*}{$\operatorname{Tm}\left[{ }^{\circ} \mathrm{C}\right]$} \\
\hline & & Material & $\begin{array}{l}\text { Hyphel } \\
\text { spores }\end{array}$ & & \\
\hline 1 & T. rubrum & skin & $++/-$ & T. rubrum & 58.73 \\
\hline 2 & T. rubrum & culture & $+1-$ & T. rubrum & 58.16 \\
\hline 3 & T. rubrum & culture & $+1-$ & T. rubrum & 58.19 \\
\hline 4 & T. soudanense & culture & n.a. & T. rubrum & 58.11 \\
\hline 5 & T. violaceum & skin & $+/-$ & T. violaceum & 68.53 \\
\hline 6 & T. violaceum & culture & $++/++$ & T. violaceum & 68.86 \\
\hline 7 & T. violaceum & skin & $++/++$ & T. violaceum & 68.62 \\
\hline 8 & T. violaceum & culture & $+++/-$ & T. violaceum & 68.91 \\
\hline 9 & T. violaceum & skin & $+++/-$ & T. violaceum & 68.72 \\
\hline 10 & T. benhamiae & culture & n.a. & T. benhamiae & 69.93 \\
\hline 11 & T. benhamiae & skin & n.a. & T. benhamiae & 69.14 \\
\hline 12 & T. benhamiae & skin & - & T. benhamiae & 69.80 \\
\hline 13 & T. benhamiae & skin & - & T. benhamiae & 70.06 \\
\hline 14 & T. benhamiae & skin & $+++/-$ & T. benhamiae & 70.15 \\
\hline 15 & T. benhamiae & skin & - & T. benhamiae & 69.90 \\
\hline 16 & T. benhamiae & skin & $+1-$ & T. benhamiae & 69.91 \\
\hline 17 & T. erinacei & culture & n.a. & T. benhamiae & 69.70 \\
\hline 18 & T. erinacei & skin & $++1-$ & T. benhamiae & 70.82 \\
\hline 19 & T. verrucosum & skin & $+1-$ & T. verrucosum & 75.77 \\
\hline 20 & T. verrucosum & skin & $+/+++$ & T. verrucosum & 75.41 \\
\hline 21 & T. verrucosum & skin & - & T. verrucosum & 75.52 \\
\hline 22 & T. verrucosum & skin & $++/-$ & T. verrucosum & 75.49 \\
\hline 23 & T. verrucosum & skin & $+++/-$ & T. verrucosum & 75.32 \\
\hline 24 & T. interdigitale & culture & n.a. & T. interdigitale & 71.44 \\
\hline 25 & T. interdigitale & culture & $++/-$ & T. interdigitale & 71.59 \\
\hline 26 & T. menta. SL & culture & n.a. & T. interdigitale & 70.83 \\
\hline 27 & T. menta. India & skin & n.a. & T. interdigitale & 72.18 \\
\hline 28 & T. menta. 1 & skin & n.a. & T. interdigitale & 72.34 \\
\hline 29 & T. menta. 1 & skin & n.a. & T. interdigitale & 71.31 \\
\hline 30 & T. quinckeanum & culture & n.a. & T. interdigitale & 72.89 \\
\hline 31 & T. quinckeanum & culture & n.a. & T. interdigitale & 73.19 \\
\hline 32 & T. menta. 2 & skin & $++/-$ & T. menta. & 78.52 \\
\hline 33 & T. menta. 2 & culture & n.a. & T. menta. & 78.70 \\
\hline 34 & T. schoenleinii & culture & n.a. & T. menta. & 78.53 \\
\hline 35 & T. tonsurans & skin & $++/-$ & T. tonsurans & 76.27 \\
\hline 36 & T. tonsurans & skin & - & T.tonsurans & 76.96 \\
\hline 37 & T. tonsurans & skin & - & T.tonsurans & 76.97 \\
\hline 38 & T. tonsurans & skin & $+1-$ & T. tonsurans & 76.38 \\
\hline 39 & T. tonsurans & skin & $-/+++$ & T. tonsurans & 76.56 \\
\hline 40 & T. equinum & culture & n.a. & T. tonsurans & 76.99 \\
\hline 41 & M. canis & skin & n.a. & M. canis & 68.09 \\
\hline 42 & M. canis & skin & - & M. canis & 68.48 \\
\hline 43 & M. canis & skin & $+++/-$ & M. canis & 68.64 \\
\hline 44 & M. canis & skin & $+/-$ & M. canis & 68.60 \\
\hline 45 & M. canis & skin & $+++/-$ & M. canis & 68.39 \\
\hline 46 & M. canis & skin & - & M. canis & 68.52 \\
\hline 47 & M. ferrugineum & culture & n.a. & M. canis & 68.72 \\
\hline 48 & M. audouinii & culture & n.a. & M. audouinii & 60.85 \\
\hline 49 & E. floccosum & culture & n.a. & E. floccosum & 69.50 \\
\hline
\end{tabular}

Seq.: sequencing, $+I-$ : presence/ absense of fungal hyphe or spores, menta.: mentagrophytes, SL: snow leopard; Tm: Melting peak temperature; n.a.: not available

\section{Material and methods}

The DNA of 31 clinically isolated skin specimens and 18 fungal cultures was isolated using the QIAamp DNA Mini Kit (Qiagen). Dermatophytes were identified macroscopically by cultural detection, partly microscopically by fluorescence staining with Blancophor ${ }^{\circledR}$ and molecular biologically using an uniplex PCR-ELISA. Sanger sequencing of both, the ITS region of the rDNA and TEF1 $\alpha$ gene was used to further analyse dermatophytes when routine diagnostics could not give a definite result (see Tab. 1). Quantitative real-time polymerase chain reaction (qPCR) was performed with the DermaGenius $^{\circledR}$ nail and complete multiplex kits and the qPCR instrument LightCycler 480 II (Roche).

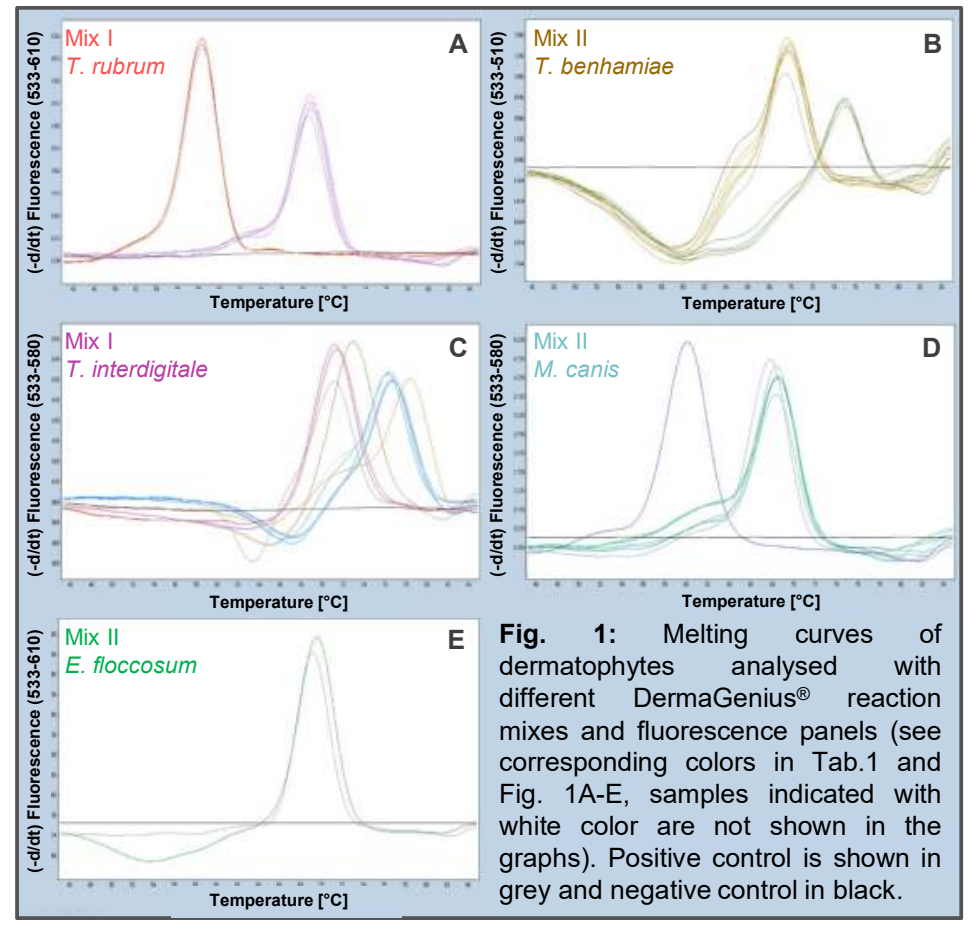

\section{Results}

In total, all $(n=49)$ clinical specimens and cultures could be detected with the DermaGenius ${ }^{\circledR}$ complete multiplex assay (PathoNostics). Out of 49 samples, DermaGenius ${ }^{\circledR}$ complete assay could identify $37(76 \%)$ correctly by their specific melting temperature (see Fig. 1). Fourteen samples were detected, but not differentiated including $T$. soudanense $(n=1)$ which was identified as $T$. rubrum, $T$. erinace $i$ $(\mathrm{n}=2)$ as $T$. benhamiae, $T$. equinum $(\mathrm{n}=1)$ as $T$. tonsurans and $M$. ferrugineum $(\mathrm{n}=1)$ as $M$. canis. Although different variants of T. mentagrophytes $(n=4), T$. quinckeanum $(n=2)$ and T. schoenleinii $(n=1)$ species were detected by qPCR, they all showed similar melting temperatures to either $T$. interdigitale or $T$. mentagrophytes.

Conclusion

The DermaGenius ${ }^{\circledR}$ qPCR assays are able to identify the most frequently isolated clinically prevalent dermatophytes and are suitable for routine molecular diagnostic laboratories as they enable high samples throughput with limited hands-on time. However, the identification and differentiation of less common dermatophytes as well as variants of $T$. mentagrophytes is still complicated since they are phylogenetically very closely related. After taking the anamnesis of patients or a fungal culture into consideration, only sequencing enables an identification of dermatophytes that could not be detected during routine diagnostics. 